\title{
A Novel Framework for Virtual Organization Creation on Cloud
}

\author{
Mona Zamanian ${ }^{1}$, Mehran Mohsenzadeh $^{1}$, and Ramin Nassiri ${ }^{2}$ \\ ${ }^{1}$ Department of Computer Engineering, Science and Research Branch, \\ Islamic Azad University, Tehran, Iran \\ $\{\mathrm{m}$.zamanian, mohsenzadeh\} @srbiau.ac.ir \\ ${ }^{2}$ Department of Computer Engineering, Central Tehran Branch, \\ Islamic Azad University, Tehran, Iran \\ r_nasiriaiauctb.ac.ir
}

\begin{abstract}
Nowadays, virtual organizations (VOs) need to utilize new technologies in order to respond rapidly to collaboration opportunities. Cloud computing is a new paradigm that could leverage VO efficiency and flexibility whilst it could decrease VO expenditures and response time. Also, VO breeding environment (VBE) is an approach to create efficient VOs. Thus, combination of VBE and cloud computing may facilitate VOs creation and reactions to upcoming opportunities. This paper has proposed a framework to create $\mathrm{VO}$ on cloud environment, including three phases: creating and managing VBE on cloud, moving VBE on cloud and finally creating VO on cloud. The proposed framework may be considered as a solution to cope with VO requirements.
\end{abstract}

Keywords: Virtual Organization, Cloud Computing, Virtual Breeding Environment, Virtual Organization Creation.

\section{Introduction}

Virtual organization (VO) is an example of collaborative networks and refers to temporary or permanent cooperation of autonomous, independent and geographically dispersed entities. However, these entities act as a unique organization and provide customers product or service[1,2]. During the recent years, VO concept is more and less combined with technology to facilitate partner's cooperation and to respond to business opportunities effectively. The first step of VO life cycle is VO creation[3] and more researchers attempt to create an efficient VO. But an important issue in VO creation is preparedness of potential partners which is neglected in most of the studies[3]. While ECOLEAD project introduces Virtual Breeding Environment (VBE) to address this issue and prevent self-interested partner's behavior[3, 4]. It integrates organizations with common principles and infrastructures to increase partner selection speed and respond rapidly to opportunities as VOs[3, 4]. This project suggested a process to create VOs from VBE with three main steps[3]: preparatory planning phase, consortia formation phase and VO launching phase. Another research[5] integrates structure of $\mathrm{VO}$ in VBE with service oriented architecture 
(SOA) and TOGAF methodology to generate Virtual Organization Breeding Methodology (VOBM). The VOBM tailors TOGAF's Architecture Development Method (ADM) to characteristics of VO and introduces ADM4VO. One early example can be found in [6] that it analyzed problems of multi-agent systems (MAS) and designed THOMAS architecture for VOs by integrating of the MAS paradigm and the service-oriented paradigm. The main problem of MAS is impracticability[3, 6] and THOMAS attempts to solve this. But ignoring of subjective facets like trust and successful cooperation history[3] is still remaining at it.

The emergence of grid and cloud computing brought a new approach for VOs and its creation, which could improve service quality. In this context, three researches can be referred: Agora[7] is a layered VO architecture for grid which it provides shared resources to grid users, CloudVO[8] and CVOE[9] that both compose a VO from cloud partners to use cloud services for resource allocation. Agora and CloudVO are special-purpose method and are employed for shared recourses whereas we intended to design a general-purpose framework. CVOE runs a business process by cloud services and assumes to provide all activities of the process by cloud services.

Among the above studies, the VO process of ECOLEAD Project has enough attention to preparedness of $\mathrm{VO}$ partners and subjective facets. Besides the process is a practical and generic process for $\mathrm{VO}$ creation. The process could be mixed with new technologies to increase efficiency and flexibility of $\mathrm{VO}$ and to decrease its expenditures. So we integrate VBE and cloud computing to exploit advantages of both and present a distinct approach for VO creation on cloud. The rest of this paper is structured as follows. Section 2 illustrates integration of VBE and cloud computing. Section 3 introduces proposed framework and their phases. Section 4 presents an example to evaluate proposed framework and finally section 5 gives conclusions.

\section{Integration of VBE and Cloud Computing}

Most of the cloud-based VO creation methods employ cloud partners alone, but the clouds only provide services and don't know VO business processes. In this paper, a VO is formed the organizations and the clouds. So that, organizations utilize cloud services to respond business opportunities and to provide customer with product or service in an effective manner. To realize this idea, firstly VBE put two different scenarios on cloud: one scenario occurs when the VBE doesn't already exist and the aim is the creation of new VBE on cloud (branch1 in Fig. 1) and other scenario moves exiting VBE on cloud (branch 2 in Fig. 1). Hence, VBE prepares organizations and clouds to collaborate each other and to create a VO on cloud when a business opportunity arises (branch 3 in Fig. 1).

This paper proposes an extended framework to achieve the objectives and facilitate VO creation on cloud by running its phases based on an existing framework[3]. It is necessary to redefine VBE and modify it with required changes. The modified definition considers the clouds as VBE partners, but they cannot undertake decisionmaking roles such as VO planner and VO coordinator. Thereafter, VBE is a long-term cooperation agreement of organizations, clouds and their related supporting 
institutions, where partners adopt its rules and operating principles. Unlike previous definition, there is no need to adopt organization's infrastructure, because it is provided by cloud services according to SOA standards. The modified VBE could prepare its partners for VOs, familiarize organizations with cloud services, build trust among them and create VOs on cloud.

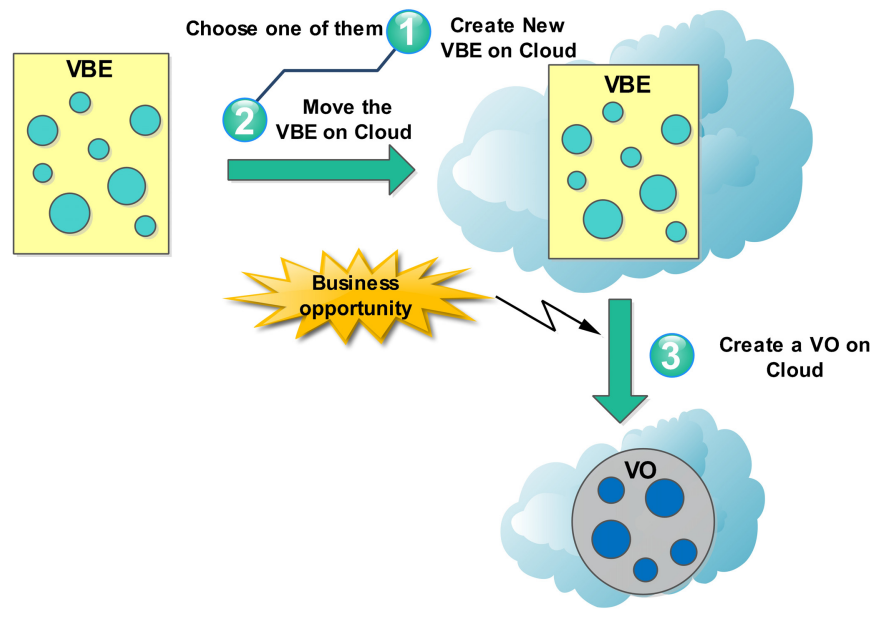

Fig. 1. Integration of VBE and cloud computing

\section{Proposed Framework}

The proposed framework is based on ECOLEAD project that is improved by three layers: business layer, service layer and component layer. Business and service layers emerge to cover two key elements, which the former is VBE organizations and the latter is cloud services. Component layer is defined to identify components of each service according to user's requirements and to provide access permissions at the component level. In fact the VBE; participating organizations and VOs use cloud services and expect to receive specific components from each service. Besides a cloud provides services in form of three models[10]: Infrastructure as a Service (IaaS), Platform as a Service (PaaS) and Software as a Service (SaaS). Granulating of the services is coarse and unsuitable to define access permissions. Thus, we define and assign permissions based on components of service.

We also define three phases, is explained in the following, to cover three branches in Fig. 1. The phases employ proposed layers to classify its activities and operations.

\subsection{Phase 1: Creating and Managing VBE on Cloud}

This phase intends to create a new VBE on cloud and manage it to support building VOs on cloud. The phase is realized at first by analyzing processes, working 
principles[11] and functionality of $\mathrm{VBE}[1]$; then classifying them according to proposed layers and defining activities to manage clouds and their services in order to deal with the cloud computing concept; finally introducing the following process to cover concepts of ECOLEAD project $[1,4,11]$ and providing a new classification according to cloud computing as:

- Business layer: the layer focuses on VBE concept, collaboration process, rules, organizations and communication. They are detailed in $[1,11]$ and are arranged in four categories now:

- Foundational concepts and rules: to declare the definition of concepts, processes, policies, working principles, ethical code and selection of supporting institutions depending on the VBE domain.

- VOs management: refers to creation and registration of VOs at the VBE and management of VO performance and VO inheritance information.

- Organizations management: refers to search, selecting and registering of new organizations as well as assigning roles and responsibilities when a VBE creates. During VBE operation, it refers to trust, competency and performance's management that may lead to rewarding or punishing organizations.

- Interactions: a VBE on cloud interacts not only with VOs but also their clouds to provide services, which are matched with user demands and have reasonable cost. The VBE interacts other VBEs to utilize their information, practices and common services stored on clouds. The VBE manages interaction between organizations and clouds to facilitate their cooperation. For example, VBE can guarantee to offer cloud services with less cost or free to organizations.

- Service layer: handles clouds and their services through the following activities:

- Clouds management: the clouds are VBE partners and should be managed, like organizations. When a VBE creates, administrator searches and selects clouds according to business layer subjects. Then selected clouds register in VBE and sign a Service Level Agreement (SLA). During the VBE operating, VBE evaluates cloud's performance, manages trust among organizations and clouds and if necessary provides incentive or penalties for them.

- Cloud services management: this activity intends to provide easy and fast cloud services. So issues to consider: identify needed services for user (VBE, its organizations and upcoming VOs) that is a prerequisite for searching clouds to guarantee proper selection; register services and update them to search services at VO creation quickly; composition of services and registering them to rapidly respond to opportunities; extract services; service orchestration according to VBE processes and finally activation of services for users.

- Information services management: the clouds maintain VBE information and provide them as services, including: VBE ontology that should be adopted with its domain at the VBE operation stage[1]; knowledge refers to initial knowledge of organizations and generated knowledge which both should be protected from unauthorized access; and other information such as competencies, services, bag of assets, VO inheritance, etc. is gathered in VBE lifecycle. The information should be inserted, updated and processed by cloud providers. 
- Other services management: cloud partners provide all of hardware and software of VBE, its organizations and ICT Infrastructure (ICT-I) by services which should be synchronized and activated at VBE startup. So the VBE supports its organization's movement toward cloud infrastructure.

- Component layer: manages components of cloud service offered to VBE user.

- Components management: in this framework, although clouds are responsible for provision, maintenance, damage and error of components, the VBE should identify expected components of each service; defines access permissions at component level and assigns the permissions to intended roles.

\subsection{Phase 2: Moving VBE on Cloud}

This phase occurs when exiting VBE would be moved on cloud. Generally, exiting VBE comprises information, systems and activities[1,11], which each of them moves on cloud as follows:

- Information: VBE information is stored in VBE repositories and actual movement of the information is nearly impossible, because it is more expensive and may cause losing or damaging information. So it offers another solution that is, making a cloud of VBE information and repositories to convert VBE into a cloud.

- Systems: VBE systems include hardware, software, ICT-I and tools are tailored with the VBE. VBE administrator could provide ICT-I, hardware and possible software of VBE and its organizations by cloud services. But, the VBE has special software and tools e.g. legacy systems, which may not be found in any clouds. They are moved like as information and are offered as services to utilize other VBEs. The solution can be used for VO creation support tools and ICT-I services[1] are developed in ECOLEAD project to save time and cost.

- Activities: VBE activities should be coordinated with the cloud computing concept. So we can use activities of prior phase and propose a process to move exiting VBE. In contrast to previous phase, the VBE exists now and have some of the activities, thus the process will create by editing previous process in the future.

\subsection{Phase 3: Creating VO on Cloud}

This phase creates VOs on cloud inside of VBE sitting on cloud now. This is done by matching VO creation process in ECOLEAD project[3] with cloud computing concept and adding new activities to it. Next the activities are classified in business, service and component layer (Fig 2.) and introduces a modern sequential process for VO creation on cloud with the same ECOLEAD steps as:

- Collaboration Opportunity $(\mathrm{CO})$ identification: this step extracts $\mathrm{CO}$ characteristics to fit one of the collaboration models: collaborative business process, collaborative project, problem solving and ad-hoc collaboration model[1].

- Rough VO planning: this step employs business, service and component layer to design VO planning and respond to CO. Business layer defines VO business structure and identifies competencies. Service layer characterizes service plan and needed services and finally, component layer specifies components of each service. 
- Partners search and selection: in general, VO planner searches VBE to select proper organizations and clouds according to rough VO planning. If VO planner doesn't find desired partners in VBE, he will explore out of VBE. This step may be repeated during VO operation to cover new needs or inefficiency of partners[3].

- Negotiation: this step is applied in most VO creation steps. In fact, it completes searching and selecting step and triggers contracting step[3]. VO planner negotiates with organizations, clouds and customer to reach agreement.

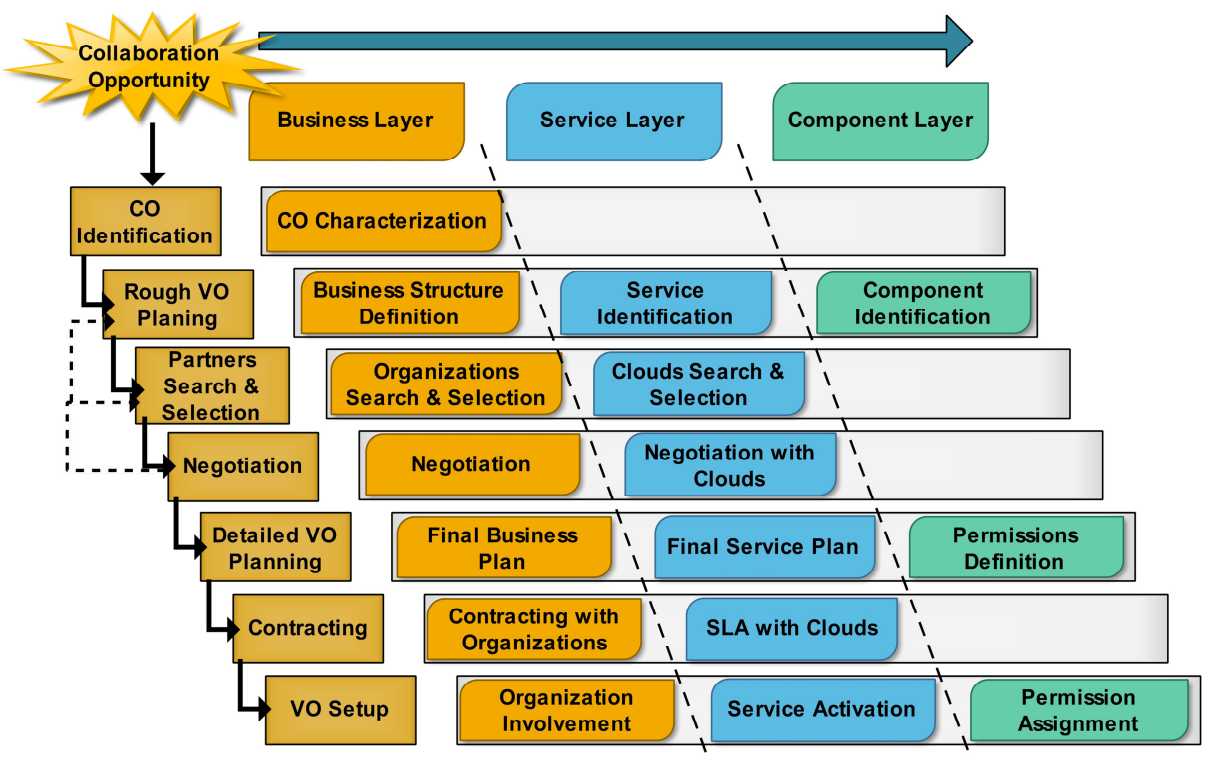

Fig. 2. Process of VO creation on cloud

- Detailed VO planning: in business layer, VO planner reviews VO business structure; forms collaboration process based on the selected collaboration model; assigns organization's role based on the process and specifies policies, working principles and VO scheduling[3]. In service layer, he improves the service plan; changes services if necessary and consequently adds or removes associated clouds. In component layer, he defines access permissions according to organization's role and collaboration process. Note access permissions can be changed or inserted during VO operation and they expire after VO dissolution.

- Contracting: addresses contracting with selected partners or in other words final organizations and clouds. Organization's contract contains responsibilities, liabilities, rights and role of organization[3]. Cloud's SLA specifies service characteristic, quality of service, accessibility, reliability and cost of service.

- VO setup: finally, VO coordinator informs organizations about VO startup time in business layer; notifies clouds to activate its services in service layer and assigns permissions to related organizations in component layer. After this step, VO enters operation phase and starts operating activities[3]. 


\section{Example}

Proposed framework is a generic framework and should be tailored with VBE domain and its VOs. This section gives an example to illustrate how to create a new VBE on cloud in order to built VO on cloud when an opportunity arises.

Hence, software development domain is selected to create a new VBE on cloud. The VBE's mission is integrating organizations competencies and cloud services to develop high quality software according to customer requests in reasonable time and cost. It executes analyzing, designing, implementing and testing processes to develop desired software. To pursue software license rights and software development's standards are its rules. The VBE employs organizations working on software development and registers their name, address, competencies, previous experiences, certificates, annual revenue and etc., then it identifies needed component and services to select cloud partners. Some VBE's components provided by IaaS e.g. data storage, processing, network and bandwidth. The VOs require various platforms and application for analysis, design, programming and testing to develop software. Other components consist of customer management application and project management application, collaboration applications, partners search and selection tools. This step specifies carefully the number of user; volume of data processing; type of operating system; database and programming language in order to identify services that may be composed of one or more components. Now VBE selects proper clouds, adjusts their SLA and registers their information including: name, type and components of service(s), accessibility, reliability, compatibility, security and cost of service(s). At this point, VBE executes activities of other service management to go operating phases and performs the rest of activities proposed to manage VBE in first phase.

A business opportunity, that is a software development's order, triggers the VO creation process on cloud. Software development is a team working project and so its collaboration model is the collaborative project. VO planner designs rough VO planning and specifies needed competencies, services and components according to the order. For example, these are different in Windows based application or UNIX based application. Next he searches and selects appropriate partners and negotiates with them to achieve a suitable configuration. Planner defines VO process and improves the service plan, assigns roles and responsibilities of organizations and defines access permission for them. Then, detailed plan is checked with customer and if he confirmed it, a contract adjusts between customer and all of VO partners. Now, VO startup is reported to all of partners; activated services; assigned permissions and registered the $\mathrm{VO}$ in $\mathrm{VBE}$.

\section{Conclusions}

The idea of VBE and cloud computing integration is proposed in this paper to create VO on cloud and to improve ECOLEAD project. The VBE prepares their organizations and clouds to collaborate upcoming VOs and so responds rapidly to business opportunities. The clouds control the components of VO to save cost and time of VO. The VBE constantly evaluates its partners (organizations and clouds) to select efficient VO partners. Also the VBE monitors partners and builds trust among them through partners management to raise VO efficiency. Cloud services of VBE 
and VO could easily be integrated by SOA standards. Furthermore the idea enhances flexibility of VO for easily replace VO partners when the customer demands changes. The cloud infrastructure allows VBE organizations to collaborate independently for time and place. Proposed framework considers subjective facets and employs the clouds beside organizations. So it could be more realistic and more common than other approaches. It employs three layers to classify activities of VBE or VO thus simplifies their management. The framework includes three phases that can be customized with different domains as shown in section 4 . The second phase of the proposed framework adopts previous studies. The cloud computing concept realizes VBE movement on cloud. We are going to accomplish this phase to offer VBE movement process in future. We also intend to develop a methodology, which is compatible with the proposed framework to describe its operation in details and to generate corresponding cloud services. Because it considers the clouds as superprovider that can provide any kind of services. Other future works include: establishing common ontology for VBEs on cloud and management of VO on cloud.

\section{References}

1. Camarinha-Matos, L.M., Afsarmanesh, H., Ollus, M.: Methods and tools for collaborative networked organizations (2008)

2. Nami, M.R.: Virtual Organizations: An Overview. In: Shi, Z., Mercier-Laurent, E., Leake, D. (eds.) Intelligent Information Processing IV. IFIP, vol. 288, pp. 211-219. Springer, Boston (2008)

3. Camarinha-Matos, L.M., Afsarmanesh, H.: A framework for virtual organization creation in a breeding environment. Annual Reviews in Control 31, 119-135 (2007)

4. Afsarmanesh, H., Camarinha-matos, L.M.: A framework for management of virtual organization breeding environments. In: Camarinha-Matos, L.M., Afsarmanesh, H., Ortiz, A. (eds.) Collaborative Networks and Their Breeding Environments. IFIP, vol. 186, pp. 35-48. Springer, Boston (2005)

5. Paszkiewicz, Z., Picard, W.: Modeling virtual organization architecture with the virtual organization breeding methodology. In: Camarinha-Matos, L.M., Paraskakis, I., Afsarmanesh, H. (eds.) PRO-VE 2009. IFIP AICT, vol. 307, pp. 187-196. Springer, Heidelberg (2009)

6. Argente, E., Botti, V., Carrascosa, C., Giret, A., Julian, V., Rebollo, M.: An abstract architecture for virtual organizations: The THOMAS approach. Knowledge and Information Systems 29, 379-403 (2011)

7. Zou, Y., Zha, L., Wang, X., Zhou, H., Li, P.: A layered virtual organization architecture for grid. The Journal of Supercomputing 51, 333-351 (2010)

8. Li, J., Li, B., Du, Z., Meng, L.: CloudVO: Building a Secure Virtual Organization for Multiple Clouds Collaboration. In: 2010 11th ACIS International Conference on Software Engineering Artificial Intelligence Networking and Parallel/Distributed Computing (SNPD), pp. 181-186. IEEE (2010)

9. Cretu, L.G.: Cloud-based Virtual Organization Engineering. Informatica Economică 16, 98-109 (2012)

10. Mell, P., Grance, T.: The NIST Definition of Cloud Computing Recommendations of the National Institute of Standards and Technology. Information Technology Laboratory (2012)

11. Afsarmanesh, H., Galeano, N., Camainha-Matos, L.M.: Characterization of key components, features, and operating principles of the Virtual Breeding Environment (2005) 